\title{
Radiation efficiencies for steel plates in water
}

\author{
M.L. Rumerman* \\ Naval Surface Warfare Center Carderock Division, Ship Signatures Department - Code 7204, 9500 MacArthur \\ Boulevard, West Bethesda, MD 20817-5700, USA
}

\begin{abstract}
Statistical Energy (or similar) Analysis may use radiation efficiencies as a means of estimating acoustic power radiated by plates in bending vibration. It is common to calculate them using uncoupled sinusoidal mode shapes of simply supported plates to account for the plate boundaries and rib stiffeners. This paper shows that, when applied to steel plates bounded by water on one side, this procedure can significantly overestimate the acoustic power radiated for frequencies below about one-eighth of the coincidence frequency. With water on both sides of the plate, the cutoff is increased to about one-fourth of coincidence.
\end{abstract}

\section{Introduction}

The exterior surfaces of many structures can be modeled as assemblies of flat plates that vibrate principally in bending and radiate acoustic energy. It may be useful or necessary to quantify the radiated power levels, but direct measurement or a calculation based on measurement of the coherent normal surface velocity can be burdensome. In 1962, Maidanik [4] introduced the idea of estimating the power radiated from a flat panel reinforced by line stiffeners and vibrating in flexure by multiplying its measured mean squared vibration velocity by a calculated panel radiation resistance. The latter quantity was estimated by first considering an otherwise homogeneous simply supported rectangular plate set in an infinite impedance baffle, and assuming it to vibrate in one of its sinusoidal mode shapes with given amplitude. Integral expressions for radiated power and radiation resistance were developed and approximately evaluated for various regimes of ratios of bending wavelength to acoustic wavelength and plate length and width to bending wavelength. By combining these modal radiation resistances with the calculated resonance modal density of the panel, the overall radiation resistance of panels in resonance could be estimated in broad frequency bands containing resonant modes. Interior reinforcing ribs were accounted for by assuming that they effectively zeroed the plate velocity along their lines of attachment and treating them as additional edges. In 1982, Leppington et al. [3] refined the evaluation of the integrals for the same idealization. The panel's radiation efficiency is defined as the radiation resistance divided by the product of panel area and characteristic impedance of the ambient medium. The radiation resistance or efficiency is also useful for incorporating radiated acoustic power as a loss mechanism in theoretical power prediction models such as Statistical Energy Analysis (SEA) and Energy Finite Element Analysis (EFEA).

There are three assumptions implicit in the application of this model of radiation resistance. The first is that the impedance of a rib attachment is so large, relative to the line input impedance of the plate, that it can be considered a line of zero plate velocity. The second assumption is that the remainder of the co-planar exterior structure in which a particular panel is located can be considered a high impedance baffle for that panel. The third is that the nature of the plate's vibration is unaffected by the reaction of the acoustic medium; that is, the fields established in the plate are those that would exist in a vacuum. This paper deals principally with the second and third assumptions, and shows that they are justified only when the fluid loading is "light", as it is for steel plates in water at sufficiently high frequencies, or in air.

*Corresponding author. Tel.: +1 301227 1704; Fax: +1 301227 1496; E-mail: RumermanML@nswccd.navy.mil. 


\section{Radiation due to line supports on infinite plates}

That the nature of acoustic power radiation by forces acting on a plate is dependent on the degree of "fluid loading" can be seen by considering a line force acting normally to an infinite plate. This problem was previously discussed by Rumerman [5]; the expression for radiated acoustic power per unit length of line force is given by Eq. (1).

$$
\Pi=\frac{F^{2}}{2 \pi} \int_{-k}^{+k} \frac{\rho \omega}{\sqrt{\left(k^{2}-\alpha^{2}\right)}} \frac{d \alpha}{\left|Z_{p}(\alpha ; \omega)+Z_{f}(\alpha ; \omega)\right|^{2}}
$$

The quantity $\mathrm{F}$ is the rms value per unit length of the line force acting on the plate, uniform along its length and varying harmonically in time with frequency $f$. The formulation is in terms of wave impedances as described by Fahy [2], in which $\alpha$ is the wavenumber corresponding to the plate coordinate perpendicular to the line of application of the force and $\omega=2 \pi f$. The quantities $Z_{p}$ and $Z_{f}$ are the wave impedances of the plate and acoustic medium, respectively, which are given by Eqs (2) and (3).

$$
\begin{aligned}
& Z_{p}(\alpha ; \omega)=-i \omega \rho_{S} h\left[1-\frac{\alpha^{4}}{k_{b}^{4}}\right] k_{b}^{4}=\frac{12 \omega^{2}}{c_{c}^{2} h^{2}} \\
& Z_{f}(\alpha ; \omega)=\frac{\rho \omega}{\sqrt{\left(k^{2}-\alpha^{2}\right)}} k=\frac{\omega}{c}
\end{aligned}
$$

In these equations, $\rho_{s}$ is the mass density of the plate material, $h$ is the plate's thickness, $c_{c}$ is the speed of compressional waves along the plate, $k_{b}$ is the wavenumber of free bending waves on the plate (in-vacuum) at the frequency of interest, $\rho$ is the mass density of the acoustic medium, $c$ is the frequency-independent sound speed in that medium and $k$ is the wavenumber of waves in the acoustic medium. The frequency dependencies of the acoustic and bending wavenumbers leads to the relationship shown in Eq. (4).

$$
\beta \equiv \frac{\rho c}{\omega \rho_{s} h}=\frac{1}{\sqrt{12}} \frac{\rho c_{c}}{\rho_{s} c} \frac{k_{b}^{2}}{k^{2}} \equiv \frac{1}{\sqrt{12}} \frac{\rho c_{c}}{\rho_{s} c} \frac{1}{\Omega}
$$

Here $\beta$ is the fluid loading parameter, large at low frequencies and small at high frequencies, and $\Omega$ is the fraction of "coincidence frequency", that frequency at which the plate's bending wave speed in-vacuum is equal to the acoustic wavespeed.

When $\Omega<\frac{1}{2}$, the value of integral in Eq. (1) can be approximated by Eq. (5).

$$
\Pi \approx F^{2} \frac{\rho}{2 \omega\left(\rho_{s} h\right)^{2}}\left(1-\frac{\beta}{\sqrt{1+\beta^{2}}}\right)
$$

The rhs of Eq. (5) may in turn be approximated by Eqs (6) and (7), according to whether the fluid loading parameter is much larger than or much smaller than unity.

$$
\begin{aligned}
& \Pi \approx F^{2} \frac{1}{2 \beta^{2}} \frac{\rho}{2 \omega\left(\rho_{s} h\right)^{2}} \beta>>1 \\
& \Pi \approx F^{2} \frac{\rho}{2 \omega\left(\rho_{s} h\right)^{2}} \beta<<1
\end{aligned}
$$

A comparison of Eqs (6) and (7) suggests that the condition $\beta \approx 1$, or perhaps $\beta=\sqrt{1 / 2}$ where the two forms are equal, is a frequency boundary at which the nature of the plate radiation changes. As the frequency decreases and $\beta$ increases towards "heavy" fluid loading, the plate becomes a much weaker radiator. Similar relations and the same conclusion result from consideration of the action of a line moment drive on the plate.

The effects of fluid loading on radiation efficiency due to a line support on an infinite plate can be examined by considering the two idealizations illustrated in Fig. 1. Each represents a fluid-loaded plate clamped along the line $x=0$; in the upper sketch, the plate is fully infinite, and in the lower it is semi-infinite, clamped along its terminating edge and extended by an infinite impedance co-planar baffle. In each representation, a free bending wave of amplitude $V_{0}$ is incident upon the clamped edge. The analysis is discussed in more length in [5]. The 
Table 1

Problem Parameters

$\rho \quad 1000 \mathrm{~kg} / \mathrm{m}^{3}$

c $\quad 1500 \mathrm{~m} / \mathrm{s}$

$\rho_{s} \quad 7800 \mathrm{~kg} / \mathrm{m}^{3}$

$c_{c} \quad 5250 \mathrm{~m} / \mathrm{s}$

h $1 \mathrm{~cm}$

L $1 \mathrm{~m}$
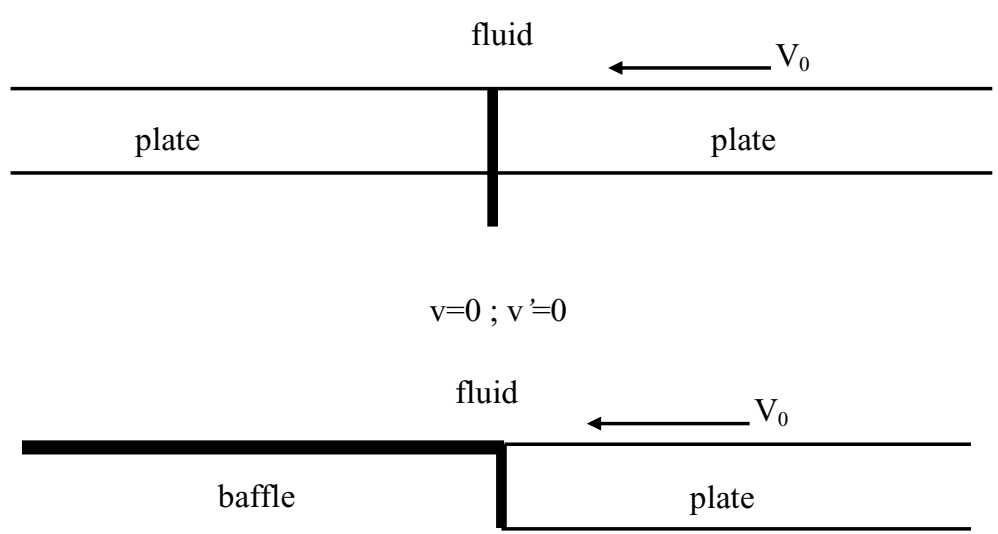

Fig. 1. Infinite plate configurations.

radiated power for the fully infinite plate is found by computing the line force and line moment input admittances for the fluid-loaded plate, determining the force and moment required to null the plate's displacement and rotation along the clamped support, and then calculating the power per unit length of support radiated by the line force and moment. The power radiated by the semi-infinite and baffled plate is found by a Wiener-Hopf analysis. A radiation efficiency $(\sigma)$ for these idealizations is defined as the power radiated per unit length of line support or edge divided by the characteristic impedance $(\rho c)$ of the ambient acoustic medium and by an arbitrary reference distance (L) in the plane of plate normal to the support.

$$
\sigma=\frac{\Pi}{\rho c L V_{0}^{2}}
$$

The calculations were performed for a steel plate with water on one side. The problem parameters are given in Table 1, and the results for the two idealizations are given in Fig. 2.

The two lowest curves represent the radiation efficiencies of the two steel plates in water. It is noted that they are approximately equal for $\Omega>0.13$, but diverge as the frequency decreases. Noting that insertion of the parameters of Table 1 into Eq. (4) yields $\beta=0.13 / \Omega$, it appears that a rigid baffle is a reasonable model for calculating radiation efficiency due to a bending wave incident upon a clamped support under light fluid loading $(\beta<1)$, but not under heavy fluid loading $(\beta>1)$. The uppermost curve represents the radiation efficiency of both the semi-infinite baffled plate and the infinite plate under "light water" loading. This fictitious fluid was given the same sound speed as that of water, so that the important ratio of free bending wavelength to acoustic wavelength was approximately maintained, but a mass density of $0.1 \%$ that of water, so that the fluid loading was very light at all frequencies considered. The single curve represents results for both the infinite plate with interior support and the baffled semi-infinite plate, because the extremely light fluid loading prevents power flow across the support through the fluid and the clamped condition blocks the structural path; therefore, the infinite plate is "self-baffling". Comparison with the other curves suggests that significant overestimates of radiation efficiency can result if proper fluid loading is omitted.

\section{Radiation efficiencies of finite width strip plates}

The analysis in this section will examine the effects of a finite dimension on the observations made for the infinite systems just considered. In order to minimize the computation required, a plate of infinite length in one direction 


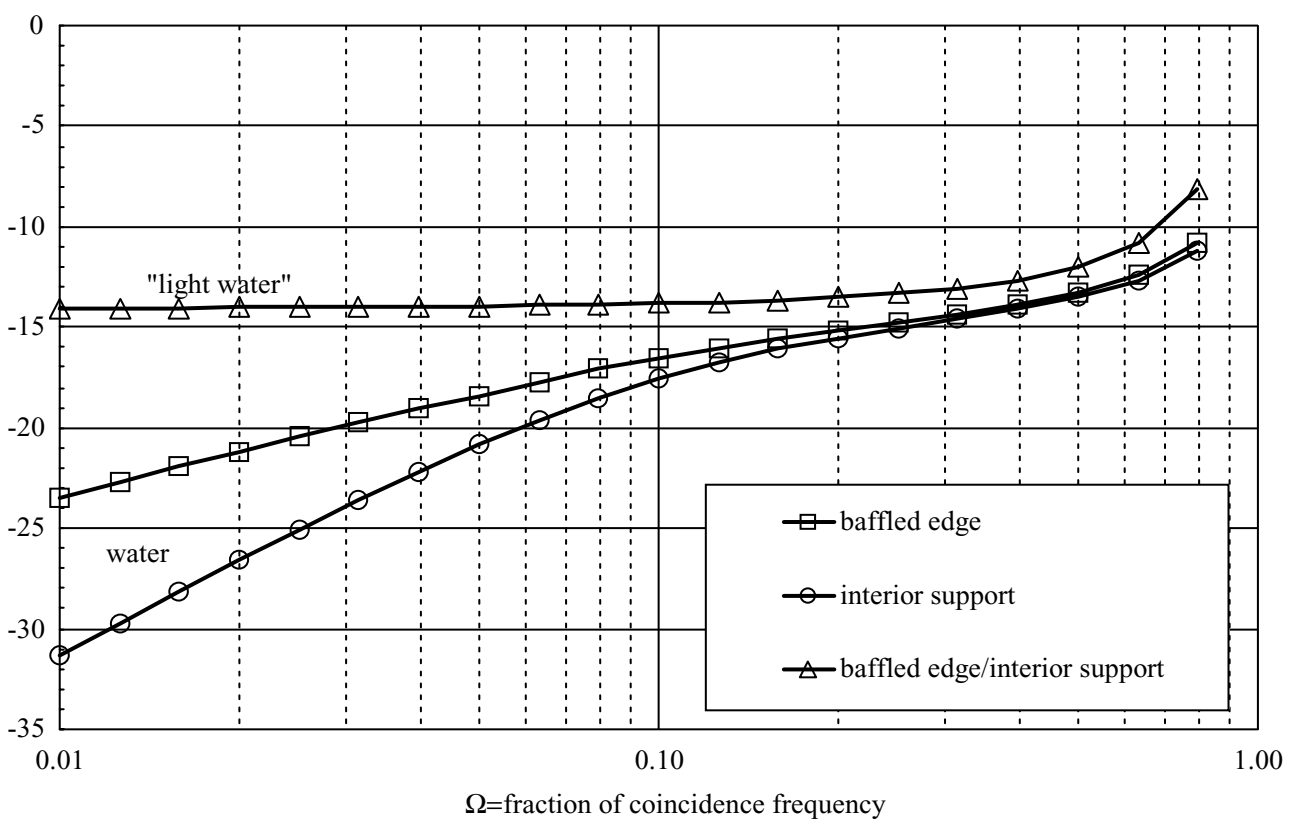

Fig. 2. Radiation efficiencies for infinite plate.

fluid

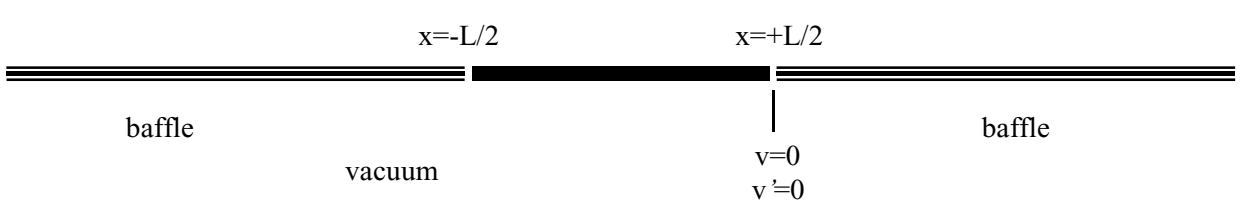

Fig. 3. Simply supported strip plate.

but of finite width in the transverse direction will be considered. Two configurations will be treated.

The first configuration consists of a strip plate placed in a co-planer rigid baffle and simply supported along the two infinite edges, as illustrated in Fig. 3. The plate is assumed to be driven by an external force per unit area, $f(x)$, that is independent of the bending velocity response $v(x)$ and induced pressure on the plate $p(x)$. A time dependence of $\exp (-i \omega t)$ is assumed but not explicitly indicated. If the excitation is restricted to be an even function of $x$, then the responses are also even, and these three quantities can be expanded in a series of basis functions $\cos \left(\alpha_{n} x\right)$, where $\alpha_{n}=(2 n-1) \pi / L$.

$$
\begin{aligned}
& v(x)=\sum_{n=1}^{\infty} V_{n} \cos \left(\alpha_{n} x\right) \\
& p(x)=\sum_{n=1}^{\infty} P_{n} \cos \left(\alpha_{n} x\right) \\
& f(x)=\sum_{n=1}^{\infty} F_{n} \cos \left(\alpha_{n} x\right)
\end{aligned}
$$

When acoustic fluid loading on the plate is light or completely absent, these basis functions are the normal modes of the plate.

The pressure on the plate is related to the velocity through the Fourier transform of the velocity. 


$$
\begin{aligned}
& \bar{v}(\alpha)=\int_{-L / 2}^{+L / 2} v(x) \exp (-i \alpha x) d x \\
& p(x)=\frac{1}{2 \pi} \int_{-\infty}^{+\infty} \bar{v}(\alpha) \frac{\rho \omega}{\sqrt{k^{2}-\alpha^{2}}} \exp (+i \alpha x) d \alpha
\end{aligned}
$$

Application of these last two equations to the series in Eq. (9) yields the pressure over the interval $-\infty<x<+\infty$ in terms of the velocity coefficients $V_{m}$, from which its projection onto any basis function may be found through another integration over $x$.

$$
\begin{aligned}
P_{n} & =\sum_{m=1}^{\infty} V_{m} Z_{m n}^{f} \\
Z_{m n}^{f} & =\frac{1}{\pi L} \int_{-\infty}^{+\infty} \frac{\rho \omega}{\sqrt{k^{2}-\alpha^{2}}}\left[\frac{2 \alpha_{m}(-1)^{m}}{\alpha^{2}-\alpha_{m}^{2}}\right]\left[\frac{2 \alpha_{n}(-1)^{n}}{\alpha^{2}-\alpha_{n}^{2}}\right] \cos ^{2}(\alpha L / 2) d \alpha
\end{aligned}
$$

Equation (14) indicates that the responses of the various basis functions are coupled and are described by Eq. (16), in which $Z_{n}^{p}$ is given by Eq. (2) with $\alpha=\alpha_{n}$.

$$
V_{n} Z_{n}^{p}+\sum_{m} V_{m} Z_{m n}^{f}=F_{n}
$$

The velocity and pressure coefficients can be calculated when the excitation is specified. The mean squared plate velocity is given by Eq. (17) and the radiated acoustic power, per unit length of the plate in the y direction, is given by Eq. (18), where * denotes complex conjugate.

$$
\begin{aligned}
V^{m s} & =\frac{1}{2} \sum_{n}\left|V_{n}^{2}\right| \\
\Pi_{\text {rad }} & =\frac{L}{2} \operatorname{Re} \sum_{n} P_{n} V_{n}^{*}
\end{aligned}
$$

The radiation efficiency is given by Eq. (19).

$$
\sigma=\frac{\Pi_{r a d}}{\rho c L V^{m s}}=\frac{R e \sum_{n} P_{n} V_{n}^{*}}{\rho c \sum_{n}\left|V_{n}^{2}\right|}
$$

In generating the results to be shown below, the plate was driven by a force distribution described by the single basis function that is expected to dominate the response at a particular frequency. Its index is given by $\left[\frac{k_{b}^{f l} L}{2 \pi}+\frac{1}{2}\right]$, where $k_{b}^{f l}$ is the free bending wavenumber for the fluid-loaded plate and the square brackets are intended to represent the nearest integer. Because the effective mass per unit area of the fluid acting on the plate is approximately $\frac{\rho}{\sqrt{k_{b}^{2}-k^{2}}} \approx \frac{\rho}{k_{b}}$ at the frequency of interest, $k_{b}^{f l}$ may be estimated as $\left(k_{b}^{f l}\right)^{4} \approx \frac{12 \omega^{2}}{c_{c}^{2} h^{2}}\left(1+\frac{\rho}{\rho_{s} k_{b} h}\right)$. Using the coupled system of Eq. (16), calculations were performed at a number of frequencies within one-third-octave frequency bands sufficient to resolve resonances within each band. The parameters are those of Table 1, except that $L=5 \mathrm{~m}$ so that there is at least one resonance in the lowest frequency band reported. The total acoustic power radiated within each band and the total mean squared velocity were found and used to calculate the radiation efficiency for each band. Results are presented in Fig. 4.

The curve with circles represents the radiation efficiency obtained using the coupled solution. The curve with triangles represents an uncoupled calculation obtained by using only the diagonal terms of $Z_{m n}^{f}$, but still summing the results for a number of frequencies within each one-third-octave frequency band. The curve with squares represents 


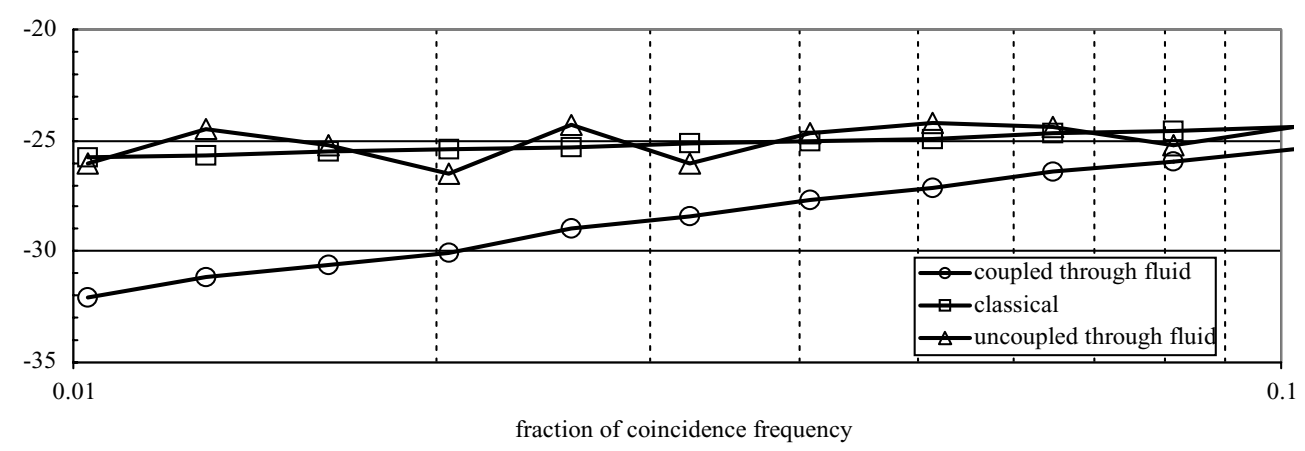

Fig. 4. Radiation efficiency of simply supported strip plate.

fluid

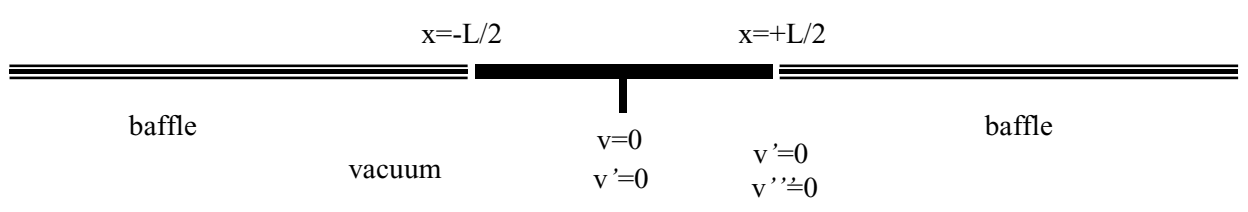

Fig. 5. Center-clamped strip plate.

the classical radiation efficiency calculated at each band's center frequency by using a single basis function, and was calculated as $\frac{\operatorname{Re}\left(Z_{n n}^{f}\right)}{\rho c}$. To obtain the real part of $Z_{n n}^{f}$ for this third calculation, $\alpha_{n}$ in Eq. (15) may be replace by $k_{b}^{f l}$ at the band's center frequency. The results again suggest that omission of the full fluid-structure interaction through the fluid coupling of basis functions, which influences wave conversion at the edges, can lead to significant overestimation of the radiation efficiency when fluid loading is "heavy".

The second configuration consists of a baffled strip plate having an infinite impedance line support at its center, as shown in Fig. 5. The edges of the strip are "quasi-free" in that the slope and shear force vanish along each edge. This allows simple basis functions to be used to represent the response, and also ensures that the radiated acoustic power is dominated by the interior support rather than the edges. Only excitation even in $x$ will be considered, so the response is also even and the plate's slope, as well as its displacement, vanishes at the support. The excitation and response are expressed by expansions similar to Eqs (9)-(11), except that $\alpha_{n}=2 n \pi / L$ and the summation begins with $n=0$

The analysis for this configuration consists of two parts. In the first, the central support is ignored and the response of the uniform plate is found. The excitation is assumed to be a force distribution described by the single basis function that is expected to dominate the response at a particular frequency. Its index is given by $\left[\frac{k_{b}^{f l} L}{2 \pi}\right]$. The second part consists of finding the force per unit length, $G$, that the support must exert on the uniform plate to null the response at $x=0$. This involves computing the line force input admittance of the uniform plate by solving Eq. (16) with $F_{n}=\varepsilon_{n} G / L ; m, n=0, \infty ; \varepsilon_{0}=1 ; \varepsilon_{n}=2, n>0$. For these basis functions, $Z_{m n}^{f}$ is given by Eq. (20).

$$
Z_{m n}^{f}=\frac{\varepsilon_{n}}{2 \pi L} \int_{-\infty}^{+\infty} \frac{\rho \omega}{\sqrt{k^{2}-\alpha^{2}}}\left[\frac{2 \alpha(-1)^{m}}{\alpha^{2}-\alpha_{m}^{2}}\right]\left[\frac{2 \alpha(-1)^{n}}{\alpha^{2}-\alpha_{n}^{2}}\right] \sin ^{2}(\alpha L / 2) d \alpha
$$

Equations (16)-(19) may be applied to the present problem if the multiplicative factor $1+\delta_{n 0}\left(\delta_{n 0}=1, n=0\right.$; $\left.\delta_{n 0}=0, n>0\right)$ is introduced into the summands of Eqs (17)-(19) to account for $n=0$. The two parts of the solution are added to yield the total plate velocity and the total surface pressure. The radiated power, mean squared velocity and radiation efficiency are then calculated as outlined above. Results are shown in Fig. 6.

The curve with circles represents the radiation efficiency obtained using the coupled solution. The curve with triangles represents an uncoupled calculation obtained by using only the diagonal terms of $Z_{m n}^{f}$, but still summing the 


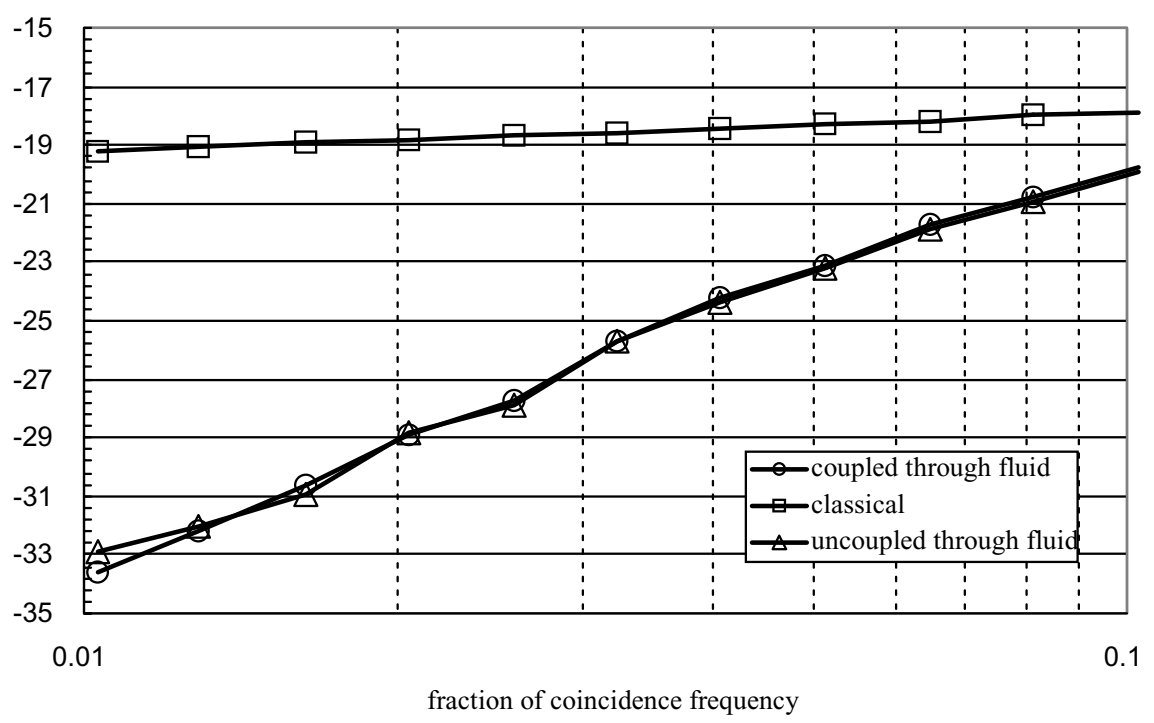

Fig. 6. Radiation efficiency of center-supported strip plate.

results for a number of frequencies within each one-third-octave frequency band. The curve with squares represents the classical radiation efficiency calculated at each band's center frequency. To properly account for the boundary conditions at the center of the plate $\left(v=0, v^{\prime}=0\right)$ and at the edges $\left(v^{\prime}=0, v^{\prime \prime \prime}=0\right)$, it is necessary to use a more complicated shape function, given by Eq. (21).

$$
\Psi_{n}(x) \approx\left[\cosh \left(\gamma_{n} x\right)-\cos \left(\gamma_{n} x\right)\right]-\left[\sinh \left(\gamma_{n}|x|\right)-\sin \left(\gamma_{n}|x|\right)\right] ; \gamma_{n} \approx(4 n-1) \pi / 2 L, n>1
$$

It is noted that $\frac{1}{L} \int_{-L / 2}^{+L / 2} \Psi_{n}^{2}(x) d x=1$. Let $\bar{\Psi}_{n}(\alpha)$ represent the Fourier transform of the shape function. The classical radiation efficiency is given by Eq. (22).

$$
\sigma=\frac{1}{2 \pi L \rho c} \int_{-k}^{+k} \frac{\rho \omega}{\sqrt{k^{2}-\alpha^{2}}} \bar{\Psi}_{n}^{2}(\alpha) d \alpha
$$

The results again indicate again that the classical radiation efficiency significantly overestimates the radiation; however, the fluid coupling of basis functions is unimportant in this case. The reason is that the radiated power is controlled, not by the edges where wave conversion is important, but by the line force exerted by the support on the plate. Even without fluid coupling, the calculation of acoustic power radiated by this force includes an adequate representation of the fluid's acoustic reaction on the plate, as in the calculation for the infinite plate.

\section{Conclusions}

Three examples of acoustic radiation from steel plates bounded by water on one side have been considered. The calculated results indicate that when the fluid loading parameter $\beta \equiv \frac{\rho c}{\omega \rho_{s} h}$ exceeds unity, the classical radiation efficiency can significantly overestimate radiated power. This corresponds to frequencies that are below about 13 percent of the coincidence frequency. If there is water on both sides of the plate, the fluid loading parameter is $\beta \equiv \frac{2 \rho c}{\omega \rho_{s} h}$ and the concern is with frequencies below about 26 percent of the coincidence frequency. If interest in radiated power level is only casual, use of the classical estimates provide a convenient upper bound. If, however, there is a not-to-exceed design objective for radiated power that must be met, an overestimate can result in unnecessary or excessive noise reduction measures and their associated costs. Care should be taken that prediction codes based on power analyses that use radiation efficiency (or an equivalent) appropriately account for fluid loading. Efforts in this direction have been reported by Blakemore and Burton [1]. 


\section{References}

[1] M. Blakemore and B. Burton, Predicting Radiation from Underwater Bodies Using AutoSEA2 - Part 1: Strong Fluid Coupling, Proceedings of the Second International AutoSEA Conference, April 2002.

[2] F. Fahy, Sound and Structural Vibration, Academic Press, Orlando, 1985, 126-136.

[3] F.G. Leppington, F.R.S. Broadbent and K.H. Heron, The Acoustic Radiation Efficiency of Rectangular Panels, (Vol. A382), Proceedings of the Royal Society of London, 1982, 245-271.

[4] G. Maidanik, Response of ribbed panels to reverberant acoustic fields, Journal of the Acoustical Society of America 34 (1962), 809-826.

[5] M.L. Rumerman, The effect of fluid loading on radiation efficiency, Journal of the Acoustical Society of America 111 (2002), $75-79$. 

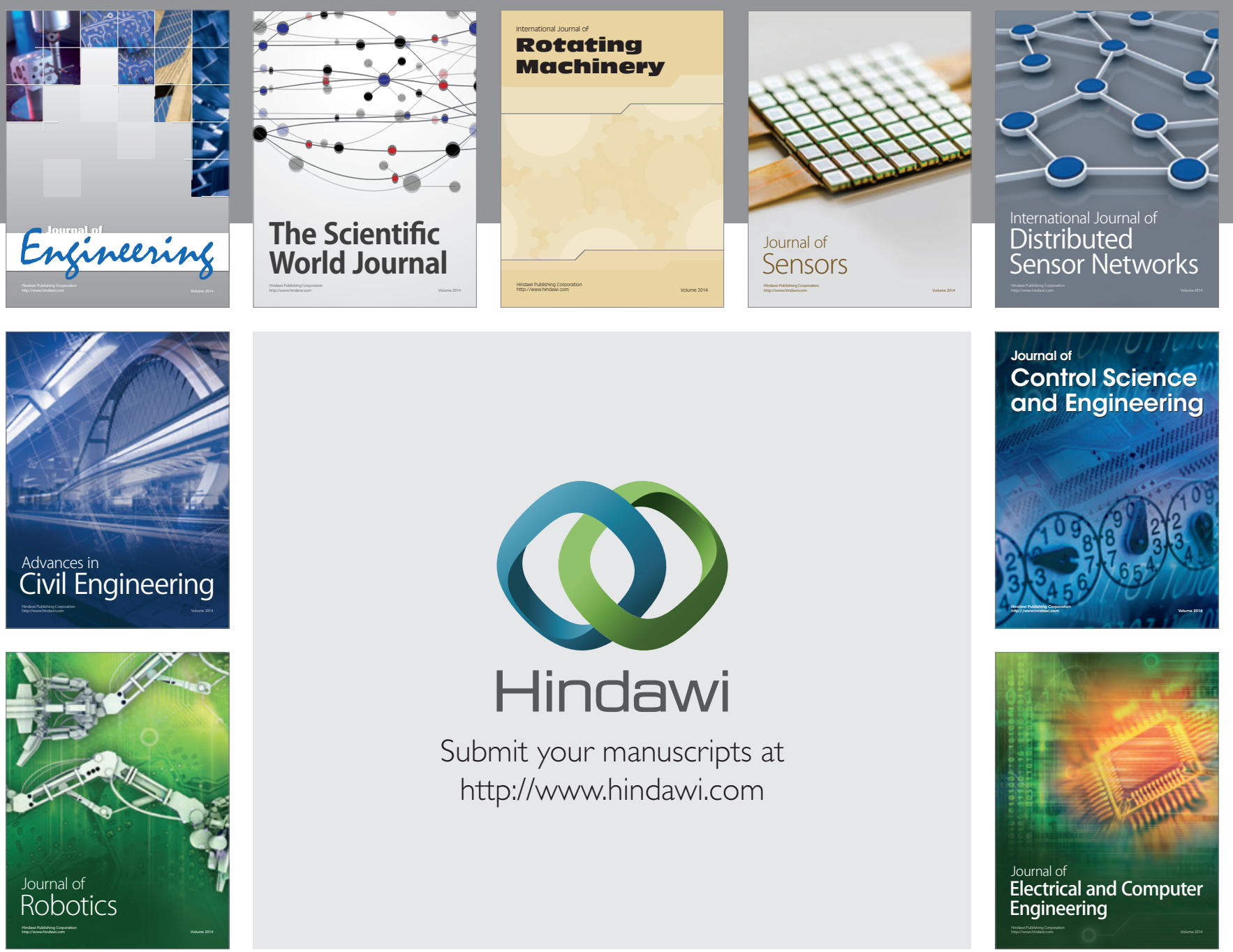

Submit your manuscripts at

http://www.hindawi.com
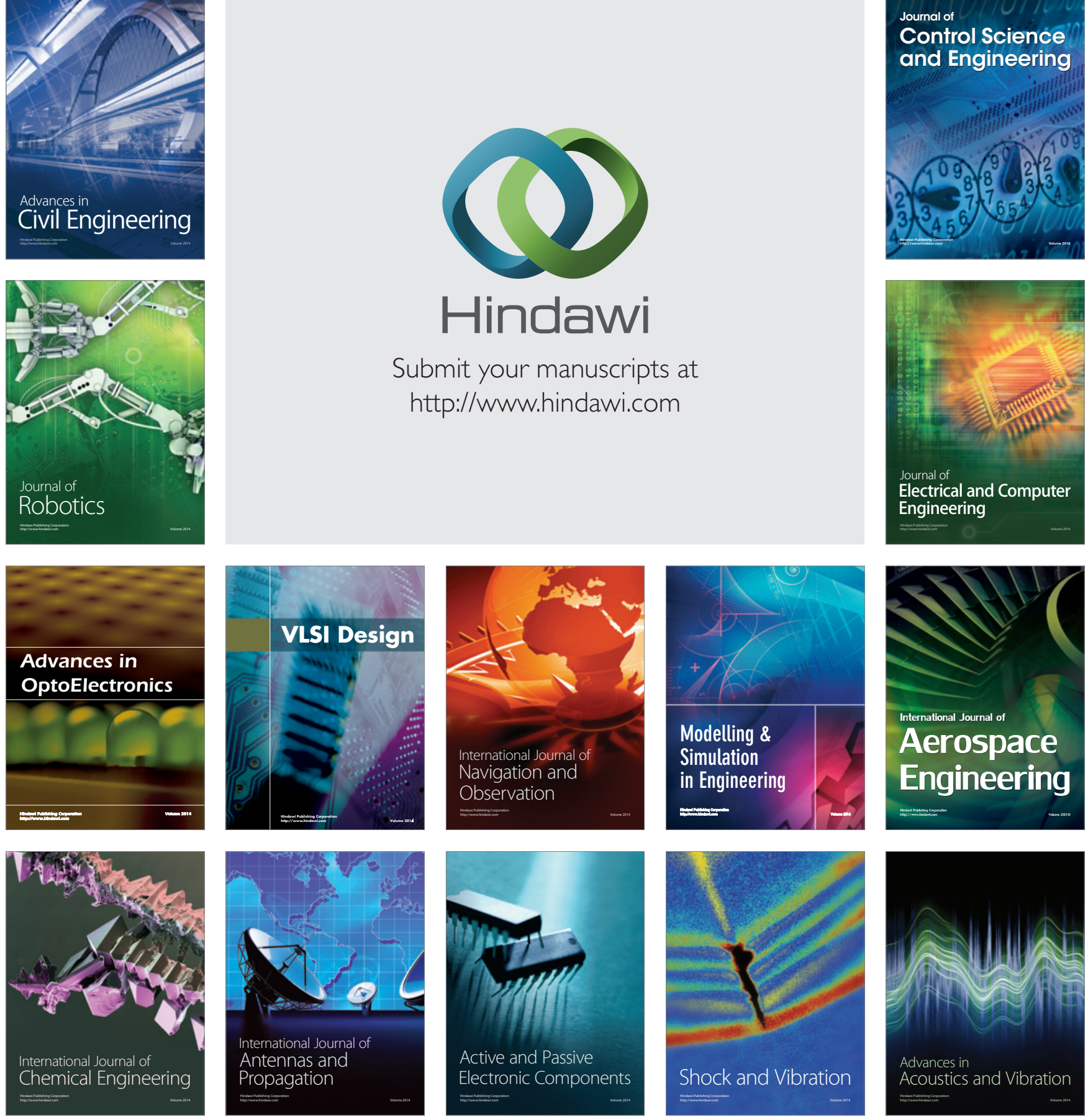